\title{
ACCURACY ASSESSMENT OF POINT CLOUDS GEO-REFERENCING IN SURVEYING AND DOCUMENTATION OF HISTORICAL COMPLEXES
}

\author{
A. Fryskowska ${ }^{a}$ \\ ${ }^{a}$ Department of Remote Sensing, Photogrammetry and Imagery Intelligence,Geodesy Institute, Faculty of Civil Engineering and \\ Geodesy, Military University of Technology, Warsaw, Poland - anna.fryskowska@ wat.edu.pl
}

KEYWORDS: Geo-referencing, Point Cloud Registration, Architectural Heritage, Accuracy

\begin{abstract}
:
Terrestrial Laser Scanning (TLS) technique is widely used for documentation and preservation of historical sites by for example creating three-dimensional (3-D) digital models or vectorial sketches. In consequence, a complex, complete, detail and accurate documentation of historical structure is created. It is very crucial when it comes about modern digital culture.

If we acquire TLS data of once particular structure usually we do it in local coordinate system of scanner. Nevertheless when measurements are conducted for complex of several historical buildings or monuments (i.e. castle ruins, building of narrow streets of the Old Towns), the registration of point clouds into a common, global coordinate system is one of the critical steps in TLS data processing. Then we have integrate data with different accuracy level. Inner accuracy of local coordinate system (scanner system) is usually thrice higher than for global coordinate systems measurement.

The paper describes the geometric quality of the direct georeferencing in post-processing, considering surveying points. Then, an analysis of factors affecting registration accuracy is proposed. Finally, an improvement of direct georeferencing technique is presented and examined. Furthermore, registered data and chosen orientation methods have been compared to each other.
\end{abstract}

\section{INTRODUCTION}

Precise documentation of historical complexes is very important for reconstruction of them when they are destroyed. Historical buildings usually have a very complex design, therefore the input data - point clouds, on the basis of which their 3D models are being built, must provide a high enough accuracy to model these complexities. Terrestrial laser scanning in spite of its costs has become a popular tool for the documentation of cultural heritage sites. Terrestrial Laser Scanning (TLS) technique is widely used for documentation and preservation of historical sites by for example creating three-dimensional digital models or vectorial sketches. In consequence, a complex, complete, detail and accurate documentation of historical structure is created. It is very crucial when it comes about modern digital culture. If we acquire TLS data of once particular structure usually we do it in local coordinate system of scanner. Nevertheless, when measurements are conducted for complex of several historical buildings or monuments (i.e. castle ruins, building of narrow streets of the old district of the city) the registration of point clouds into a common, global coordinate system is one of the critical steps in TLS data processing (Kedzierski et al., 2015) and (Wilinska et al., 2012).

Georeferencing can be done using direct orientation considering scanners with mounted GPS antenna or/and compass or in the post-processing with the use of surveying ground control points and transformations (Scaioni, M., 2005) Both solutions are charged with some errors. The importance of the accuracy of navigational data is presented in (Przyborski M., and Pyrchla J., 2003) or (Waggot et al. 2005).

This paper presents approach to assess and improve quality of direct georeferencing of terrestrial laser scanner (TLS) point clouds using modified surveying points.

\section{RELATED WORKS}

Several methods of direct georeferencing of point clouds acquired with stationary laser scanner exist. First, we can integrate terrestrial laser scanner with GPS antenna and compass like in (Böhm i Haala, 2005). The TLS has been integrated with a low accuracy GPS receiver $(2 \mathrm{~m})$ and a realtime, high accuracy $6^{\circ}$ electronic compass in the highly urbanized terrain, enabling global cloud points georeferencing accuracy up to $2 \mathrm{~m}$ (height) and $3 \mathrm{~m}$ (horizontal coordinates). Additionally, Iterative Closest Point ICP was used to improve the accuracy of the overall georeference.

Other option is georeferencing using previously designed and measured points of the geodetic network. For the purpose of scanning, six points of the GNSS measurement network using the ASG-EUPOS system were located around the object. The coordinates of the points were determined with a precision of 3 $\mathrm{cm}$. Scans were obtained from free stations, with the target planes. Authors have reached the accuracy of the building model of $14 \mathrm{~cm}$. The analysis was based on check points derived from independent tachymetric measurements. (Borkowski and Jozkow, 2012).

Another option is to use mobile laser scanning rather than static TLS measurements. This approach has been realized by, among others, the research team (Mikrut et al., 2016) or (Redstall et al., 2010) on the example of a mobile laser scanning system StreetMapper. This approach is simple because it does not require a transformation between the coordinate systems. Usually, data can be obtained directly in the global coordinate system. Both sensors (mobile and airborne laser scanner) are integrated with GPS and IMU. Authors depend on the accuracy of the final data from the place of measurement and the accuracy of the navigation systems. A similar method is also presented in other publications: (Becker and Haala, 2007), where the intensity of the point cloud and the linear features of the objects were also used, (Alshawa et al., 2009), (Haala et al., 2008) or (Frueh and Zakhor, 2003) - using terrestrial images and airborne laser scanner).

Other techniques rely on data integration. That means, georeferencing using other geodata already registered in global coordinate system (i.e. airborne laser scanning - Fryskowska et al., 2015) 


\section{MATERIALS AND METHODOLOGY}

\subsection{Problem statement and methodology}

Reference points coordinates acquired in scan registration process have to be determined in global coordinate system (GNSS measurements). When scanning for example building complex (about $1 \mathrm{sq}$. $\mathrm{km}$ of its area) number of reference points is quite large. The accuracy of reference points is about $30 \mathrm{~mm}$ for $\mathrm{X}, \mathrm{Y}$ coordinates and $50 \mathrm{~mm}$ for $\mathrm{Z}$ coordinate. Of course this accuracy is much lower when measurements are conducted for example in the narrow streets of the Old Town of the city.

For high-resolution laser scanning measurements the accuracy of reference points is about $3-5 \mathrm{~mm}$ for $\mathrm{X}, \mathrm{Y}$ and $\mathrm{Z}$ coordinates. That gives possibility to gain the inner accuracy and coincidence of laser scanning registered point clouds is about 3$8 \mathrm{~mm}$. Nevertheless, when we transform registered in LCS point clouds we decrease the accuracy to $40-60 \mathrm{~mm}$. This phenomenon can be named as the accuracy transfer between the primary and the secondary coordinate systems. In this case, the transfer will result in decreasing the accuracy of high resolution data at the georeferencing stage.

With small buildings areas we do not notice a problem with the so-called crossover/overlapping scans, but with large objects, when we scan long linear objects (i.e. streets of the old town or tunnels), this problem will decrease of the accuracy of the final product. The general problem of assessing the accuracy of this type of point cloud is the lack of reliable check points in the orientation/registration process.

The problem of the accuracy transfer is briefly presented in the figure 1 .

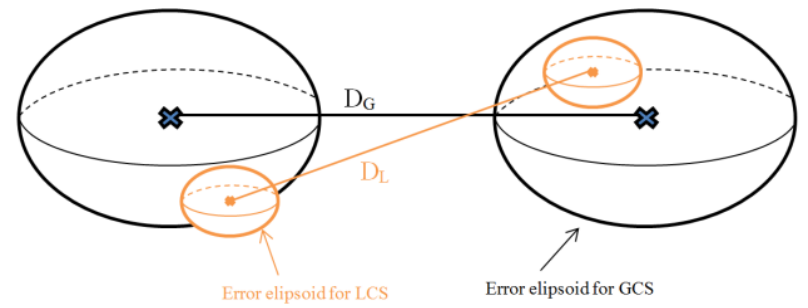

Figure 1. Objects selected for the research purpose - accuracy transfer schema.

We consider two independent systems: primary system - local coordinate system - scanner system (LCS) and secondary system - global system (GCS). The global system is charged with certain errors and by its nature is less accurate than the local system in which the point clouds were obtained. Error ellipsoid of GCS is much higher than LCS and have some particular influence on the final georeferencing accuracy. That means, that if we need to fit a point cloud into a global system, the scanned object take over errors of global system (GCS). During the registration process we are unable to eliminate these errors directly. In practice it is done using coarse and fine registration but still we do not improve the quality of basic reference points.

But we can ask the question: How to improve the accuracy of reference points (surveying points)?

One of the solutions is to correct coordinates of a the surveying reference points based on local coordinate system. Correcting global coordinate system coordinates can be done in several ways: applying transformation or alignment of the trilateration network.

In this paper only improvement by transformations is examined. In research both isometric and affine transform have been used.
Research structure was divided into two main streams:

1.At the beginning the article describes a method for assessing the accuracy of point clouds registered in global systems based on specially arranged checkpoints that are not involved in the process of orientation. This method should always be used with this type of solutions, and that this result should be taken as a measure of the accuracy.

2.In the second part of the article the author propose a method of improvement of geo-referencing process of point clouds of historical complexes. The proposed method introduces corrections to the coordinates of reference points. This is on the basis of transformation parameters.

\subsubsection{Transformations}

For isometric transformation we do not introduce scale factor. There is only translation and rotation required to calculate the coordinates of secondary system (B) on the basis of primary system (A). Isometric 3D transformation (6 parameters) is realized:

$$
\left[\begin{array}{l}
X_{B} \\
Y_{B} \\
Z_{B}
\end{array}\right]=\left[\begin{array}{l}
X_{A} \\
Y_{A} \\
Z_{A}
\end{array}\right]+\left[\begin{array}{l}
\Delta X \\
\Delta Y \\
\Delta Z
\end{array}\right]+\left[\begin{array}{ccc}
1 & \kappa & \theta \\
-\kappa & 1 & \omega \\
\theta & -\omega & 1
\end{array}\right] \cdot\left[\begin{array}{l}
X_{A} \\
Y_{A} \\
Z_{A}
\end{array}\right]
$$

where:

$\Delta \mathrm{X}, \Delta \mathrm{Y}, \Delta \mathrm{Z}$ - translation vector between means of both coordinate systems $[\mathrm{m}]$,

$\omega, \theta, \kappa$ - differences in orientation of XYZ cartesian axes between systems.

For affine transformation, the scale factor plays a role. Affine transform is a linear transformation of three-dimensional space through 3 translation parameters, 3 rotational parameters, 3 scaling parameters and 3 angular distortion parameters of the three pairs of axis of the system. As a result of affine transformation, straight lines are transformed as simple, and their parallelism is preserved. The location, orientation, shape and size of the sections change. Then, the rotation matrix can be represented as:

$$
\begin{aligned}
& R_{x}(\omega)=\left[\begin{array}{ccc}
1 & 0 & 0 \\
0 & \cos \omega & \sin \omega \\
0 & -\sin \omega & \cos \omega
\end{array}\right] \\
& R_{y}(\theta)=\left[\begin{array}{ccc}
\cos \theta & 0 & -\sin \theta \\
0 & 1 & 0 \\
\sin \theta & 0 & \cos \theta
\end{array}\right] \\
& R_{z}(k)=\left[\begin{array}{ccc}
\cos k & \sin k & 0 \\
-\sin k & \cos k & 0 \\
0 & 0 & 1
\end{array}\right]
\end{aligned}
$$

Therefore, this transformation can be problematic in some particular cases.

For the trilateral solution corrections are calculated based on the distance between the reference points acquired from point clouds before registration. The idea of the method involves treating the reference points as points of spatial geodetic network. Depending on the distribution of the reference points geometric conditions are established and the process of special alignment is carried out.

\subsection{Materials}

The method has been verified on the 800 meters long street of the old district of the city. This studies have been conducted 
using phase-based terrestrial laser scanner (Faro Focus 3D) and registration algorithms, as non-contact methods of creating building documentation.

Figure $2 \mathrm{a}$ and $2 \mathrm{~b}$ persents the coarse-registered scan of the part of the street.

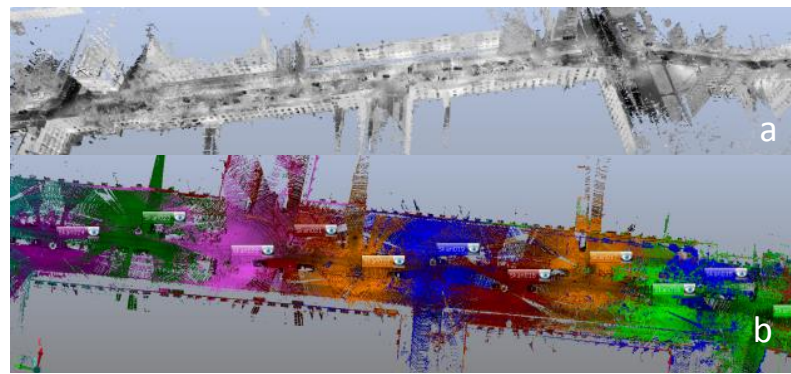

Figure 2. Registered point clouds of the part of the street in a) grey-scale b) with visible separate stations (colored)

For point cloud processing common registering algorithms have been used: target-to-target and cloud-to-cloud. The target-totarget method consists of determining the transformation parameters of one scan to another using at least three reference points selected by the operator automatically. Usually as a targets sphere, plane targets and checkerboards are used. Figure 3 presents the examples of target planes used in the experiment.

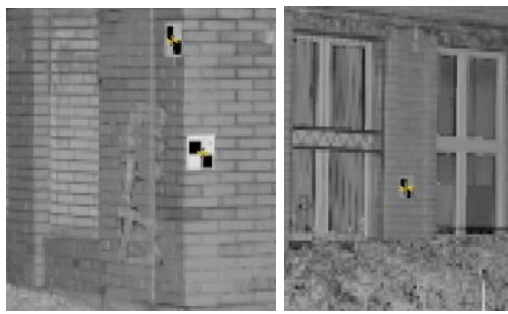

Figure 3. Checkerboard as a targets used in the experiment

Scanning was conducted in a 41 stations with 160 targets with known coordinates of global coordinate system. The accuracy of the reference points were approx. $5 \mathrm{~cm}$ and scan resolution 1$2 \mathrm{~cm}$. Not all of the targets were used as the reference points in registration. Some of them were outliers (eliminated in the preprocessing), some of them were used as check points.

The cloud-to-cloud method registers scans by matching the point clouds. Then, usually a greater number of homologous points are needed so that the least squares method could be applied in order to decrease the transformation errors. It is based on performing an estimation of the transformation parameters for one or a number of points/planes or surfaces in relation to a reference data using a generalized Gauss-Markoff model, minimizing the sum of the squares of the Euclidean distances between the surfaces. This process is done iteratively and requires at least two reference points for an initial registration. One of the methods of automatic homologous point recognition is Iterative Closest Point algorithm, described widely for example in (Chen and Medioni, 1991), (Menq and Lai, 1992), (Zhang, 1994), (Bae and Lichti, 2004) or (Rabbani et al., 2007).

\section{EXPERIMENT AND RESULTS}

\subsection{Preliminary accuracy assessment}

For the initial/preliminary analysis of the accuracy of the data obtained by laser scanning, some check measurements have been used.
The aim of this research was to determine:

1. the accuracy of stationary laser scanners point clouds measured in the local co-ordinate system.

2. Relative accuracy of local systems versus mobile laser scanning data, where georeference is determined in real-time.

The same we can answer the question if we are able to transform point clouds from LCS into GCS with comparable accuracy to mobile laser scanning measurements?

To answer this question these data were compared.

The distance between selected points measured by the tachymetric method (accuracy 3-5 mm) and the length of other elements of the object such as window openings, stairs, etc. with measuring tape (accuracy 1-2 $\mathrm{mm}$ ) were determined. The points defining the check distances selected for analysis had to meet the criterion of unambiguity and identification. Control distances were based on points measured in the local system using the TOPCON GPT3005, and the length of the sections measured with surveying tape.

An alternative method to perform point clouds georeferencing in flight is mobile laser scanning. Although this technique have some economical limitations and impossibility to conduct measurement in hard-to-reach historical sites and complexes as well.

In research Trimble MX8 mobile scanning platform was also used. The system is equipped with GPS / INS navigation devices. The range of the test area on which the segments were measured was chosen to avoid the impact of the transformation error, so that they could be identified on all data sets without the need to merge data from different scan stations. The selected sections were located in different planes and directions and were of varying length and "availability" from the perspective of selected measurement techniques. The points on which the lengths of the sections were determined were easy to identify. Table 1 contains the lengths of selected sections of the front elevation of the test building, based on tachymetric (T), pulsed (SI), phase (SP) and mobile laser scanning systems, as well as the absolute difference values of the length of the sections relatively to the tachymetric measurements (i.e. $|\mathrm{T}-\mathrm{SI}|$ ).

\begin{tabular}{|c|c|c|c|c|c|c|}
\hline $\mathbf{T}$ & SI & SP & MLS & $\mid$ T - SI $\mid$ & $\mid$ T-SP $\mid$ & $\mid$ T - MLS $\mid$ \\
\hline 1.298 & 1.314 & 1.318 & 1.195 & 0.016 & 0.020 & $\mathbf{0 . 1 0 3}$ \\
\hline 1.317 & 1.326 & 1.344 & 1.281 & 0.009 & 0.027 & $\mathbf{0 . 0 3 6}$ \\
\hline 3.198 & 3.144 & 3.167 & 3.140 & $\mathbf{0 . 0 5 4}$ & $\mathbf{0 . 0 3 1}$ & $\mathbf{0 . 0 5 8}$ \\
\hline 9.416 & 9.399 & 9.443 & 9.489 & 0.017 & 0.027 & $\mathbf{- 0 . 0 7 3}$ \\
\hline 3.793 & 3.785 & 3.792 & 3.726 & 0.008 & 0.001 & $\mathbf{0 . 0 6 7}$ \\
\hline 4.844 & 4.866 & 4.859 & 4.754 & 0.022 & 0.015 & $\mathbf{0 . 0 9 0}$ \\
\hline 4.834 & 4.863 & 4.862 & 4.788 & 0.029 & 0.028 & $\mathbf{0 . 0 4 6}$ \\
\hline 13.766 & 13.734 & 13.790 & 13.801 & $\mathbf{0 . 0 3 2}$ & 0.024 & $\mathbf{- 0 . 0 3 5}$ \\
\hline 2.342 & 2.353 & 2.351 & 2.340 & 0.011 & 0.009 & 0.002 \\
\hline
\end{tabular}

Table 1 The lengths of test sections and absolute values of length differences of selected sections in relation to tachymetric measurements

On the basis of the analysis, it can be seen, that the biggest differences between the tachymetric and the scanning measurements characterized the MLS data. This is related both to the average distance between the points in the cloud and to the navigation system errors. For the other two systems the average difference was about $2 \mathrm{~cm}$. Differences greater than the highest $3 \mathrm{~cm}$ value are bolded and are mainly characteristic for MLS data. In addition, a comparative analysis of the selected 
façade lengths of a building measured with a measuring tape (measurement accuracy 1-2 $\mathrm{mm}$ ) was performed. The analyzed elements are stairs and window openings located in different parts of the building. The absolute values of the differences between tape measurements and pulse and phase ground scanner data reached $2.5 \mathrm{~cm}$ and $1.6 \mathrm{~cm}$ values respectively, which confirms the accuracy presented in table 2. Most of these elements are objects in the lower part of the building that have not been measured by a mobile laser scanner (e.g. ground, side stairs).

\subsection{Registration accuracy}

In the experiment registration was conducted in three variants: in local coordinate system (LCS), global coordinate system using rare surveying points (GCS) and global coordinate system with the reference points acquired by $3 \mathrm{D}$ transformations (GCS affine and GCS isometric).

As it is presented in results below, not every transformation is suitable. Table 2 presents the results of registration based on procedures mentioned above.

\begin{tabular}{|l|c|c|c|c|}
\hline $\begin{array}{c}\text { Error } \\
\text { (chekerboard) }\end{array}$ & LCS & GCS & $\begin{array}{c}\text { GCS } \\
\text { Isometric }\end{array}$ & $\begin{array}{c}\text { GCS } \\
\text { Affine }\end{array}$ \\
\hline min. [m] & 0,007 & 0,009 & 0,008 & 0,007 \\
\hline max. [m] & 0,329 & 0,031 & 0,013 & 0,033 \\
\hline mean [m] & 0,019 & 0,017 & 0,011 & 0,019 \\
\hline $\begin{array}{l}\text { standard } \\
\text { deviation [m] }\end{array}$ & 0,011 & 0,010 & 0,006 & 0,011 \\
\hline
\end{tabular}

Table 2. Errors of registration using varied reference data

Coordinates of reference points were improved by corrections to rare surveying points. It was done by transforming these points from local - primary into global - secondary coordinate system. In this paper new, improved by transformation global system will be named GCS ${ }^{\mathrm{T}}$.

Transformation error of isometric transform was $0.138 \mathrm{~m}$, and for the affine $0.110 \mathrm{~m}$. These though small discrepancies are related to some differences in transformation schema. For further analysis, isometric transformation were used. The accuracy of these processes was determined on the points that did not take part in the orientation of scans, and more precisely at the distances between these points. The differences for the same check lengths between orientation in different systems reached values: between LCS and GCS: $3 \mathrm{~cm}$, between LCS and $\mathrm{GCS}^{\mathrm{T}}: 2 \mathrm{~cm}$.

In general, on the basis of research dependency have been derived. The ratio of errors of LCS registration ( $\mathrm{m}_{\mathrm{LCS}}$ ) to the error of GCS registration ( $\mathrm{m}_{\mathrm{GCS}}$ ) should be close to 1 . This would be ideal solution. That would means, that both systems have similar or the same accuracy and do not distorts none of them:

$$
\frac{m_{L C S}}{m_{G C S}} \cong 1
$$

Nevertheless usually this ratio is in the range $0.15-0.20$. That means global coordinate systems accuracy is approximately few times lower than local scanner coordinate system. The main influence gives GCS. Therefore it needs improvement. As we know, the accuracy of new, improved GCS is in the function of GCS, LCS and transformation accuracy:

$$
m_{G C S}=f\left(m_{G C S}, m_{L C S}, m_{T}\right)
$$

As it is presented in table 2, transformation gave improvement about $40 \%$. After registration scan into $\mathrm{GCS}^{\mathrm{T}}$ the ratio (3) was equal 0.3 .

\section{CONCLUSIONS}

As a result of the application of this method the improvement of the geo-referencing accuracy was by about $40 \%$. The time needed to process the new method is relatively small and can be ignored. Thanks to the proposed method, there were no errors so-called ghosting (for example double edge on the details of the building's edges, roofs). Such errors are very common and occur for historical building complexes registered in the global coordinate systems. The research showed, that through modification of geo-referencing process we can obtain accurate 3D models (point clouds) of historical complexes.

Further research will focus on other methods of laser scanning point clouds georeferencing improvements.

\section{ACKNOWLEDGEMENTS}

This paper has been supported by the Military University of Technology, the Faculty of Civil Engineering and Geodesy, Department of Remote Sensing, Photogrammetry and Imagery Intelligence.

\section{REFERENCE}

Alshawa M., Grussenmeyer P., Smigiel E., 2009. A low cost TLS based land mobile mapping system assisted by photogrammetry, Boletim de Ciencias Geodésicas, Vol.15,no5

Bae Kwang-Ho, Lichti D., 2004. Automated registration of unorganised point clouds from terrestrial laser scanners, International Archives of Photogrammetry and Remote Sensing, Vol. XXXV, Part B5, Proceedings of the ISPRS WG V/2

Becker S., Haala N., 2007. Combined feature extraction for facade reconstruction, ISPRS Workshop on Laser Scanning 2007 and SilviLaser 2007, ISPRS, Volume XXXVI

Borkowski A., Jóźków G., 2012. Ocena dokładności modelu 3D zbudowanego na podstawie danych skaningu laserowego przykład zamku piastów śląkich $w$ Brzegu, Archiwum Fotogrametrii, Kartografii i Teledetekcji, Vol. 23, 2012, s. 3747 ISSN 2083-2214 | ISBN 978-83-61576-19-8

Böhm J, Haala N., 2005. Efficient integration of aerial and terrestrial laser data for virtual city modeling using lasermaps, Proceedings of the ISPRS Workshop Laser scanning 2005, ISPRS Archives, Volume XXXVI, Netherlands, str. 192-197

Chen,Y., Medioni, G.,1991. Object modeling by registration of multiple range images, IEEE International Conference on Robotics and Automation, vol. 3, str. 2724-2729, 1991

Frueh Ch., Zakhor A., 2003. Constructing 3D City Models by Merging Aerial and Ground Views, IEEE Computer Graphics and Applications, Vol. 23, No. 6. (2003), str. 52-61

Fryskowska A., Walczykowski P., Delis P., Wojtkowska M., 2015. ALS and TLS Data Fusion In Cultural Heritage Documentation And Modeling, 25TH INTERNATIONAL CIPA SYMPOSIUM 2015, International Archives of the Photogrammetry Remote Sensing and Spatial Information Sciences, Vol. 45, Issue: W7, Pages: 147-150, DOI: 10.5194/isprsarchives-XL-5-W7-147-2015 
Haala, N., Peter, M., Cefalu, A., Kremer, J., 2008. Mobile lidar mapping for urban data capture, VSMM 2008 - Conference on Virtual Systems and MultiMedia Dedicated to Digital Heritage, Limassol, Cyprus, October 20th - 25th, 2008, str. 101-106

Kedzierski M., Fryskowska A., Wierzbicki D., Dabrowska M., Grochala A., 2015. Impact Of The Method Of Registering Terrestrial Laser Scanning Data On The Quality Of Documenting Cultural Heritage Structures, International Archives of the Photogrammetry Remote Sensing and Spatial Information Sciences Vol. 45 Issue: W7 Pages: 245-248 DOI: 10.5194/isprsarchives-XL-5-W7-245-2015

Menq Yau H.-T.C.-H., Lai G.-Y., 1992. Automated precision measurement of surface profile in cad-directed inspection, IEEE Trans. RA. 8(2), str. 268-278,

Mikrut S., Kohut P., Pyka K., Tokarczyk R., Barszcz T., Uhl T., 2016. Mobile Laser Scanning Systems for Measuring the Clearance Gauge of Railways: State of Play, Testing and Outlook, Sensors, Vol. 16, Issue: 5, DOI: 10.3390/s16050683

Przyborski M., and Pyrchla J., 2003. Reliability of the navigational data, Intelligent Information Processing And Web Mining, Advances In Soft Computing, Pages: 541-545

Rabbani T., Dijkman S., van den Heuvel F., Vosselman G., 2007. An integrated approach for modelling and global registration of point clouds, ISPRS Journal of Photogrammetry and Remote Sensing, Volume 61, Issue 6, February 2007, str. 355-370

Redstall M., Grierson H., Duffell C.,2010. Fusion of mobile terrestrial and airborne laser scanning data, Conference Proceedings

Scaioni, M., 2005, Direct Georeferencing of TLS in Surveying of Complex Sites, The International Archives of the Photogrammetry, Remote Sensing and Spatial Information Sciences (IAPRS), Vol.36, Part 5/W17

Waggot, S.M., Clegg, P., Jones, R.R., 2005. Combining Terrestrial Laser Scanning, RTK GPS and 3D Visualisation: Application of Optical 3D Measurement in Geological Exploration, Proceedings of the 7th Conference on 3-D Optical Measurement Techniques, 3-5 Oct., Austria

Wilinska M., Kedzierski M., Zaplata R., Fryskowska A., Delis P., et al., 2012. Noninvasive methods of determining historical objects deformation using TLS, Structural Analysis Of Historical Constructions, vol: 1-3, Pages: 2582-2588

Zhang Z., 1994. Iterative point matching for registration of free-form curves and surfaces, International Journal of Computer Vision, 13(2), str. 119-152, 